\title{
Географическое изучение «умных городов»: приоритеты, подходы, перспективы
}

\author{
В.И. Блануца \\ Институт географии им. В.Б. Сочавы СО РАН, Российская Федерация \\ (664033, г. Иркутск, ул. Улан-Баторская, 1)
}

\begin{abstract}
Аннотация: Цель: выявить существующие подходы и наметить контуры будущих подходов к комплексному географическому изучению «умных городов».

Информационной базой служат одна отечественная и семь международных библиографических баз данных.

Memodbl: сравнительный анализ и авторский алгоритм семантического поиска на основе машинного обучения.

Результаты и обсуждение. Выявлена 91 статья. Показано распределение статей по странам и масштабам исследования. Анализ статей позволил идентифицировать восемь существующих подходов: сравнительно-географический, пространственно-урбанистический, районный, геополитический, пространственно-сегрегационный, темпорально-ландшафтный, пространственно-диффузионный и геосистемный. Сделано предположение, что в будущем могут появиться еще три подхода: позиционно-географический, потоковый и системно-расселенческий. Относительно небольшое количество статей указывает на стадию становления рассматриваемых исследований. В дальнейшем возможна конвергенция всех подходов в единую методологию географического изучения «умных городов».
\end{abstract}

Ключевые слова: социально-экономическая география, умный город, сравнительный анализ, районирование, социально-пространственное неравенство, пространственная диффузия, экономико-географическое положение.

Источник финансирования: Исследование выполнено за счет средств государственного задания (№ регистрации темы АААА-А17-117041910166-3).

Для цитирования: Блануца В. И. Географическое изучение «умных городов»: приоритеты, подходы, перспективы // Вестник Воронежского государственного университета. Серия: География. Геоэкология, 2021, № 3, c. 3-12. DOI: https://doi.org/10.17308/geo.2021.3/3595

\section{ВВЕДЕНИЕ}

Объектом нашего исследования является мировой опыт географического изучения «умных городов». Под «умным городом» понимается территориально распределенное множество сенсоров и других цифровых устройств, объединенное с помощью «Интернета вещей» и генерирующее «большие данные», которые обрабатываются искусственным интеллектом и другими алгоритмами для принятия оптимальных решений по управлению развитием города.

Цель работь - выявить существующие подходы и наметить контуры будущих направлений к комплексному географическому изучению «умных городов». Основные задачи: идентифи-

(C) Блануца В.И., 2021

$\triangle$ Блануца Виктор Иванович, e-mail: blanutsa@list.ru цировать мировой массив журнальных статей с результатами эмпирических исследований по рассматриваемой проблематике; определить приоритеты географических исследований, результаты которых зафиксированы в отобранных статьях; свести мировое разнообразие способов и предметов исследования к небольшому количеству общегеографических подходов; наметить контуры возможных новых направлений.

\section{МАТЕРИАЛЫ И МЕТОДЫ}

Первые разработки по использованию информационно-коммуникационных технологий в управлении городами появились в 1970-х годах в рамках концепции «городской кибернетики» [19]. Дальнейшее развитие этих идей привело

(c) (i) Контент доступен под лицензией Creative Commons Attribution 4.0 License. 
к созданию в начале 1990-х годов концепции «умного города» («Smart City») [23, 31]. В качестве отдельных блоков выступали «умная экономика», «умная инфраструктура», «умная окружающая среда», «умные горожане», «умная жизнь» и «умное управление» [18]. Несмотря на фрагментарность и тематическую разнонаправленность исследований, что характерно для новых областей познания, появились обобщения публикаций по «умным городам» в рамках различных научных дисциплин [23, 36, 43]. Однако данная тенденция не затронула социально-экономическую географию. При этом отмечалось, что «умный город» является «пространственно включенным городом» [42], обладает «пространственным интеллектом» [39] и представляет «последовательные доказательства географии городов» [10].

Информационная база. Для возможности идентификации подходов и территориальной привязки результатов, полученных при изучении «умных городов», рассматривались только публикации с новыми эмпирическими данными комплексного характера. Частные случаи (например, «умный транспорт» или «умный туризм») не анализировались, так как это выходило за рамки общей социально-экономической географии (к примеру, относилось к географии транспорта или рекреационной географии). Из всех видов публикаций (монографии, сборники статей и др.) выбраны журнальные статьи, поскольку в международных библиографических базах данных только по всем статьям имелись полные тексты. Для выяв- ления мирового массива статей использовались одна отечественная и семь международных баз данных - www.elibrary.ru, www.link.springer.com, www.onlinelibrary.wiley.com, www.sciencedirect.com, www.login.webofknowledge.com, www.scopus.com, www.journals.sagepub.com, www.ideas.repec.org.

Memodbl. Для отбора статей применялся авторский алгоритм семантического поиска публикаций по заданной теме, позволяющий в базе данных в автоматическом режиме находить необходимые статьи на основе машинного обучения [4]. При настройке параметров семантического поиска были заданы ограничения, удаляющие не географические исследования, частные географические работы и географические публикации без эмпирических результатов. Далее в отобранных статьях устанавливались сходства и различия с помощью сравнительного анализа.

\section{РЕЗУЛЬТАТЫ И ОБСУЖДЕНИЕ}

С помощью семантического поиска среди публикаций 1990-2020 годов удалось выявить 91 статью. Первые географические печатные работы по проблеме «умных городов» приходятся на 1999-2008 годы. В 1999 году в географическом журнале впервые упоминается «умный город» Сингапур [34]. В 2007 году препринтом публикуются результаты географического изучения «умных городов» Центром региональной науки Венского технологического университета, где сравнительному географическому анализу были подвергнуты 70 средних городов Европы [18]. И только в 2008 году появляется

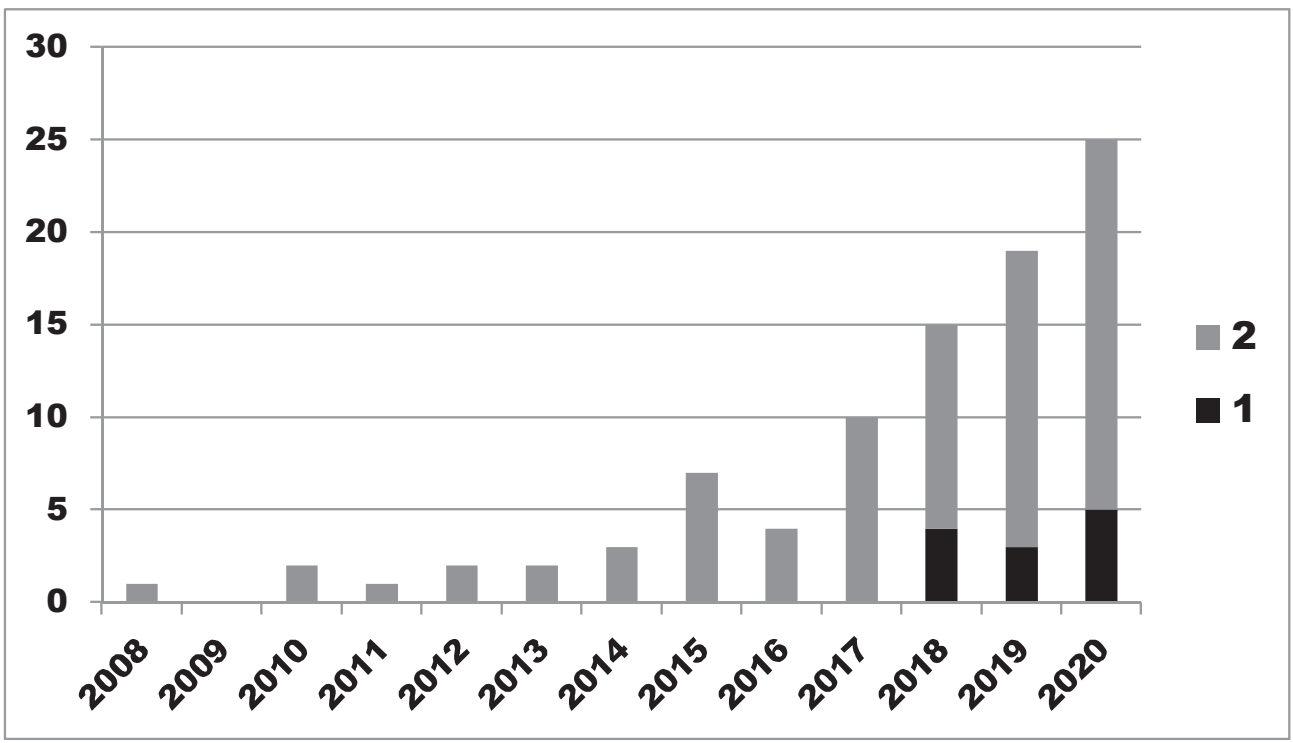

Puc. 1. Изменение ежегодного количества статей по географическому изучению «умных городов», опубликованных в географических (1) и других (2) научных журналах во всем мире в 2008-2020 годах (составлено автором)

[Fig. 1. Change in the annual number of articles on the geographical study of «smart cities» published in geographical (1) and other (2) scientific journals worldwide in 2008-2020 (compiled by the author)] 
в свет первая журнальная статья, посвященная географическому анализу «умных городов» [22].

Первое время отмечается небольшое количество статей с эмпирическими результатами комплексных географических исследований «умных городов». Но с 2017 года происходит их существенное увеличение (рис. 1). Выявленные статьи представлены в 62 журналах, среди которых выделяются «Cities» и «Sustainability» (по 8 статей). По одной статье опубликовано в 12 географических журналах.

Среди стран мира наибольшее количество статей поступило в журналы из Великобритании - 13,65 (при наличии авторов из разных стран статья как единица счета делилась на число стран), а более трех статей приняты еще из 10 стран (рис. 2). Географическим изучением «умных городов» занимаются исследователи из 30 стран, среди которых большинство (20 стран) расположено в Европе (66,15 статей). Что касается распределения изученных городов по частям света, то больше всего их приходится на Европу (56,35 статей), Азию $(21,74)$ и Америку $(10,08)$. При учете пяти масштабов исследования (от локального, связанного с отдельным городом, до глобального, охватывающего все «умные города» в мире) следует отметить, что больше всего публикаций посвящено межнациональному (45 статей) и национальному (37) масштабам. Не очень востребованными оказались региональный (5 статей), локальный (4) и глобальный (0) масштабы исследования.

В каждой проанализированной статье использовался определенный авторский стиль к географическому изучению «умных городов», но все они могут быть объединены в восемь общегеографических подходов.

Сравнительно-географический подход (71 статья). Представляет собой сопоставление различных территорий (частей города, городов, групп городов) по определенному набору показателей (параметров) с целью выявления некоторых географических особенностей (закономерностей). В отобранном массиве статей сравнение проводилось двумя способами. Первый способ сводился к определению набора параметров «умного города», введению критерия «умности» («smartness») по каждому параметру и выделению «умных городов» среди анализируемых поселений $[35,48]$. Второй заключался в создании образа «умного города» (эталонной модели) как определенного сочетания значений различных параметров и проведении проверки на соответствие этому образу городов, которые по политическим или экономическим мотивам уже объявили себя «умными городами» [22, 46]. В этих сравнительных исследованиях создавались «пространственно-временная модель интеллектуального развития городов» [12], многомерные индексы [24], «нейронная сеть

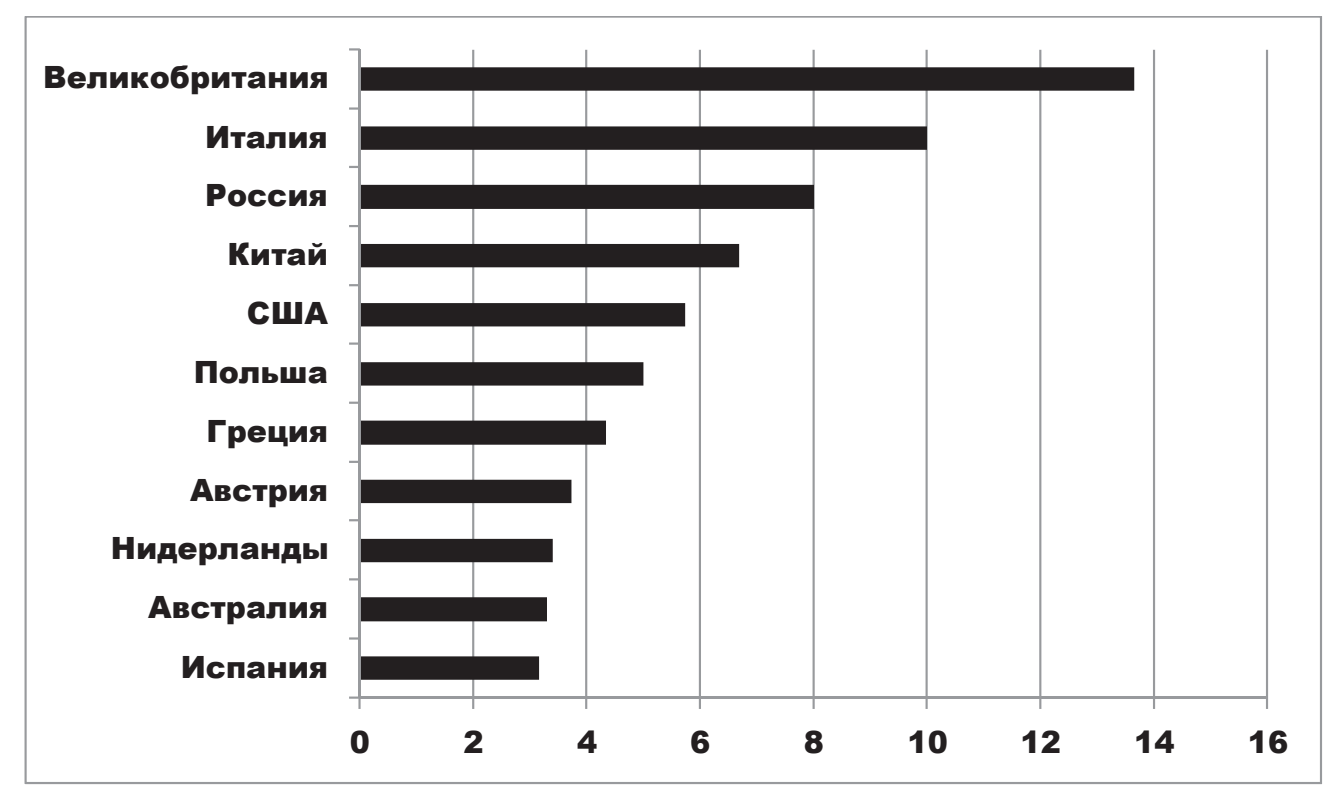

Рис. 2. Ранжирование стран по количеству опубликованных статей (не менее трех статей в 2008-2020 годах; отнесение к стране по аффилиации автора), посвященных географическому изучению «умных городов» (составлено автором)

[Fig. 2. Ranking of countries by the number of published articles (at least three articles in 2008-2020; assigned to the country by the author's affiliation) devoted to the geographical study of «smart cities» (compiled by the author)] 
самоорганизующейся карты» [28], типология городов [40], «индекс демократичности умного управления» [33].

В качестве основного результата сравнительного анализа чаще всего предлагался рейтинг городов. Так было заложено в пионерной работе австрийских исследователей [18]. В дальнейшем эти наработки с различными модификациями использовались для ранжирования городов по степени «умности», например, в Европе [8], Румынии [17], Сербии [48] и Польше [35]. К другим результатам сравнения можно отнести формулировку вариантов стратегического выбора политики развития «умных городов» [6], отбор наилучших практик [24], оценку взаимосвязи между «умностью» города и его людностью [8], пространственные характеристики современного развития сети городов (например, «глянцевитость, фрагментарность и хаотичность» [49]) и характер реагирования на пандемию COVID-19 [16].

Дальнейшее развитие подхода может быть связано со следующими векторами: 1) заменой универсального набора показателей «умного города» на специфические сочетания параметров, более характерные для отдельных стран и их групп (например, уже предложена «северная система измерения» для оценки арктических городов [41]); 2) переходом от сопоставления статичных «умных городов» к сравнению траекторий их развития; 3) расширением классификации городов по степени «умности» до территориально-структурной типологии, когда выявляется внутригородское распределение плотности «умных» объектов и сравнение городов происходит по этим структурам; 4) смещением акцентов исследования с разработки системы показателей «умности» для ранжирования городов на идентификацию и сравнение внешних сетевых эффектов, обуславливающих ускоренную трансформацию обычного города в «умный город»; 5) ориентацией на выявление местных социально-экономических факторов, способствующих развитию одних и препятствующих росту других «умных городов» (одна из таких попыток затронула только два города - Ванкувер и Сиэтл [21]).

Пространственно-урбанистический подход (7 статей). Основная идея заключается в распространении инициативы «умный город» на другие виды населенных пунктов и межселенное пространство. Так появились концепции «умного пространства» [27], «умного региона» [32] и «умной деревни» [47]. Стало больше внимания уделяться сельской периферии [45] и городским окраинам [14]. При переходе от «умного города» к урбанизированному пространству предлагалось развивать «умную специализацию» территории [11]. В качестве примера реализации подхода можно привести исследование по муниципалитетам Бельгии [15]. Нерешенные проблемы и формируемые на их основе векторы дальнейших исследований повторяют предыдущий подход. Кроме этого, необходимо изучать региональные и межнациональные различия между параметрами «умного города» и «умной территорией», а также разработка методологии делимитации таких территорий, так как в настоящее время границы не выявляются, а привязываются к административно-территориальному делению.

Районный подход (5 статей). Районирование - один из основных способов выявления целостных и специфических территориальных образований (районов) в социально-экономической географии [2]. Применительно к «умным городам» существует проблема выявления их территориальных скоплений, в которых происходит интенсивное взаимодействие городов. Один из вариантов решения проблемы - выделение цифровых («умных») городских агломераций по сверхмалой задержке сигнала в оптоволоконной сети [3]. Подобная проблема существует и внутри отдельно взятого города. На примере индийского города Бхопал продемонстрировано [37], что для планирования развития «умного города» необходимо разделить городское пространство на ячейки и провести их «пространственную интеграцию» в территориальные образования с «низким, средним и высоким приоритетом». Основные проблемы подхода были рассмотрены ранее [2], а перспективы связаны с анализом «больших данных» по «умным городам» и выявлением искусственно-интеллектуальных районов, в которых происходит концентрация и взаимодействие производственных, транспортных и сервисных автономных систем искусственного интеллекта.

Геополитический подход (4 статьи). Использование технологий создания «умного города» для закрепления одних и отделения других проблемных территорий составляет основу данного подхода. Примером может служить анализ реализации «Миссии умных городов» в «чувствительном пространстве» северо-востока Индии [30], согласно которому главной целью миссии в этой части страны является интеграция пограничного пространства в национальную территорию. Противоположное направление связано с концепцией 
«умной деволюции» (передача полномочий по созданию «умных городов» от центрального правительства к местной власти), которую предлагается [9] использовать в Глазго (Великобритания), Барселоне и Бильбао (оба города в Испании) с целью создания «умных» городов-регионов в Шотландии, Каталонии и Стране Басков с последующим их отделением. В связи с ограниченностью публикаций по данному подходу в перспективе возможно много векторов развития географических исследований.

Пространственно-сегрегаиионный подход (1 статья). Технократический способ управления «умным городом» активно критикуется с разных позиций $[5,22]$. Проблема в том, что реализация инициативы «умный город» приводит к новому социально-пространственному неравенству в городах. Результатом этого стало формирование «критической школы» [29] познания такого неравенства и изучение «географии алгоритмического насилия» («повторяющаяся и стандартизированная форма насилия, которая способствует расизации пространства и пространственной бедности» [44, p. 200]). Однако изучение новой сегрегации в городах проводится в основном социологами. Социально-экономической географии еще предстоит разработать методологию эмпирических исследований социально-пространственной сегрегации внутри «умного города» [5]. Из опыта анализа разных территорий можно привести пример по малым городам штата Айова (США), в которых выявлена «умная усадка» (депопуляция при сохранении высокого качества жизни определенных слоев населения) [38].

Темпорально-ландмафтный подход (1 статья). Географическое изучение «умных городов» опирается на пространственно-временной анализ, который в большинстве случаев слабо учитывает хронологическую составляющую. Особенно это проявляется на локальном уровне. Для устранения данной диспропорции предложено идентифицировать темпоральные (временные) ландшафты в «умном городе». На примере Дублина показано [26], что «умный город» формирует новый временной ландшафт в результате сжатия пространства-времени и создания множества специфически локализованных городских ритмов. Сделан вывод о том, что именно изменение темпа повседневной деятельности во временных ландшафтах является основным преимуществом «умного города», а не реконфигурация пространственных отношений. Дальнейшие исследования могут быть направлены на выявление темпоральных «умных» ландшафтов на территории вне городов, формирование таксономической системы таких ландшафтов и создание темпорально-ландшафтных карт.

Пространственно-диффузионный подход (1). Начиная с работ Т. Хегерстранда [20], в географии уделяется внимание анализу пространственной диффузии инноваций. Однако по рассматриваемой проблематике имеется только одно эмпирическое исследование, посвященное внедрению технологий «умного города» в органах местного самоуправления в Южной Корее [25]. Положительная пространственная автокорреляция, выявленная в этом исследовании, указывает на наличие диффузионного процесса распространения интеллектуальных технологий. Перспективы подхода связаны с переходом от модели контагиозной диффузии к выявлению иерархической и сетевой пространственной диффузии [3], определением направленности диффузионных волн, а также возникающих на их пути барьеров и фильтров.

Геосистемный подход (1 статья). «Умный город» можно представить в виде единой цифровой экосистемы «Интернета вещей», но с географических позиций более целесообразно оперировать множеством взаимодействующих геосистем. Такая возможность заложена в проекте «Умного Дублина», но картографирование этого города [13] остается более экосистемным, чем геосистемным. Развитию подхода могут способствовать создание концепции «умной городской геосистемы», обоснование таксономических единиц и разработка алгоритма выделения геосистем.

Исследования «умных городов» в рамках социально-экономической географии начались относительно недавно, и все перечисленные выше подходы требуют теоретического обоснования, совершенствования алгоритмов и развернутой апробации. В этом смысле в перспективе необходимо дальнейшее развитие восьми выделенных нами подходов. Однако в географии накоплен определенный методологический опыт изучения других объектов, который до настоящего времени не использовался при анализе «умных городов». Поэтому целесообразно наметить новые контуры, которые могут быть реализованы в будущем. По мнению автора, наибольшую значимость имеют три будущих направления.

Позииионно-географическое. Изучение экономико-географического положения (ЭГП) городов - отличительная черта отечественной школы экономической географии [1]. С учетом расшире- 
ния исходных представлений об ЭГП, связанных с развитием сетевого (ЭГП как взаиморасположение сетей) и потокового (ЭГП как степень вовлечения в системообразующие потоки) направлений [7], открывается возможность познания экономико-географического положения «умных городов». На конкретных примерах предстоит уяснить, как ЭГП города способствует или препятствует его превращению в «умный город». Не исключено, что наработок в области изучения сетевого положения будет недостаточно и потребуется концептуализация нового вида ЭГП - геоинтеллектуального положения. Дополнительными направлениями в рамках данного подхода могут быть изучение ЭГП «умных городов» как интеграционного потенциала, вектора и дрейфующего центра [7].

Потоковое. Потоки информации между городами являются предметом изучения социально-экономической географии [3]. Однако до настоящего времени не проводились географические исследования потоков «больших данных» между «умными городами». С одной стороны, такие исследования будут способствовать развитию предыдущего подхода (ЭГП как степень вовлечения в потоки), с другой - позволят оценить интенсивность взаимодействия «умного города» с окружающими обычными городами и другими «умными городами». От потоковых моделей можно будет перейти к построению балансовых и гравитационных моделей, а это значительно расширит географическое понимание «умных городов».

Системно-расселенческое. «Умные города» еще не получили повсеместного распространения, что не позволяет оценить их воздействие на систему расселения на региональном, национальном и глобальном уровнях. Однако уже имеются отдельные не взаимосвязанные исследования потенциального воздействия технологий «умного города» на малые [38], средние $[18,48]$ и крупнейшие [50] города. Но эти работы не нацелены на комплексныйанализвсех вариантов воздействия на систему расселения как единое целое. Поэтому предстоит разработать методологию географического изучения трансформации систем расселения в результате распространения «умных городов», а также провести эмпирические исследования для апробации методологии и определения сфер применения полученных результатов.

\section{ЗАКЛЮЧЕНИЕ}

С помощью специального алгоритма семантического поиска в библиографических базах данных обнаружен ряд статей, посвященных комплексным эмпирическим исследованиям «умных городов» в рамках социально-экономической географии. Анализ статей показал, что рассматриваемые исследования находятся в стадии становления, о чем свидетельствуют относительно небольшое количество статей (91), запоздалое (относительно других дисциплин) появление первой статьи (2008 г. по сравнению с 1990 г.) и доминирование публикаций по географическому сравнению городов (около $80 \%$ ). Вне сравнительного анализа выявлено семь подходов. Сделано авторское предположение, что в будущем могут появиться еще три новых направления. В дальнейшем, вероятно, наступит стадия консолидации эмпирических исследований и произойдет конвергенция разных подходов в единую методологию географического изучения «умных городов». Предпосылки для такого предположения уже существуют. Они связаны с накоплением данных в результате географических сравнений и подключением к этому процессу территорий вне городов, дополненное углубленным изучением временных ландшафтов, «умных» геосистем и социально-пространственной сегрегации. Во-вторых, происходит перераспределение информационных потоков, пространственной диффузией инноваций и изменением экономико-географического положения. Все это приведет к более полному пониманию формируемых интегральных районов и их воздействию на систему расселения и принятие геополитических решений.

\section{СПИСОК ЛИТЕРАТУРЫ}

1. Баранский Н.Н. Избранные труды. Становление экономической географии. Москва: Мысль, 1980. 287 с.

2. Блануца В. И. Социально-экономическое районирование в эпоху больших данных. Москва: ИНФРА-М, 2018. $194 \mathrm{c}$.

3. Блануца В.И. Информаиионно-сетевая география. Москва: ИНФРА-М, 2019. 243 с.

4. Блануца В.И. Региональные экономические исследования с использованием алгоритмов искусственного интеллекта: состояние и перспективы // Becmник Забайкальского государственного университета, 2020 , т. 26 , № 8, c. $100-111$. DOI: $10.21209 / 2227-9245$ 2020-26-8-100-111.

5. Adam S. A critical geographical analysis of the smart city concept - Theoretical background and possible research directions // Ter es Tarsadalom, 2020, vol. 34, no. 2, pp. 88-107. DOI: 10.17649/TET.34.2.3201.

6. Angelidou M. Smart city policies: A spatial approach // Cities, 2014, vol. 41, pp. S3-S11. DOI: 10.1016/j. cities.2014.06.007.

7. Blanutsa V.I. Economic-geographical location: Generalization of conceptual frameworks and generation of new meanings // Geography and Natural Re- 
sources, 2015, vol. 36, no. 4, pp. 319-326. DOI: 10.1134/ s1875372815040010.

8. Borsekova K., Koróny S., Vaňová A., Vitálišová K. Functionality between the size indicators of smart cities: A research challenge with policy implications // Cities, 2018, vol. 78, pp. 17-26. DOI: 10.1016/j.cities.2018.03.010.

9. Calzada I. Problematizing and politicizing smart city-regions: Is devolution smart? // Territorio, 2017, vol. 83, pp. 37-47. DOI: 10.3280/TR2017-083005.

10. Caragliu A., Del Bo C., Nijkamp P. Smart cities in Europe // Journal of Urban Technology, 2011, vol. 18, no. 2, pp. 65-82. DOI: 10.1080/10630732.2011.601117.

11. Caragliu A., Del Bo C. Much ado about something? An appraisal of the relationship between smart city and smart specialization policies // Tijdschrift voor Economische en Sociale Geographie, 2018, vol. 109, no. 1, pp. 129-143. DOI: 10.1111/tesg.12272.

12. Chen X., Wei L., Zhang H. Spatial and temporal pattern of urban smart development in China and its driving mechanism // Chinese Geographical Science, 2018, vol. 28, no. 4, pp. 584-599. DOI: 10.1007/s11769-018-0976-0.

13. Coletta C., Heaphy L., Kitchin R. From the accidental to articulated smart city: The creation and work of "Smart Dublin" // European Urban and Regional Studies, 2019, vol. 26, no. 4, pp. 349-364. DOI: $10.1177 / 0969776418785214$.

14. De Falco S., Angelidou M., Addie J.-P.D. From the "smart city" to the "smart metropolis"? Building resilience in the urban periphery // European Urban and Regional Studies, 2019, vol. 26, no. 2, pp. 205-223. DOI: $10.1177 / 0969776418783813$.

15. Desdemoustier J., Crutzen N., Giffinger R. Municipalities' understanding of the Smart City concept: An exploratory analysis in Belgium // Technological Forecasting and Social Change, 2019, vol. 142, pp. 129-141. DOI: 10.1016/j.techfore.2018.10.029.

16. Fariniuk T.M.D. Smart cities and the pandemic: Digital technologies on the urban management of Brazilian cities // Revista de Administração Pública, 2020, vol. 54, no. 4, pp. 860-873.

17. Georgescu M., Tugui A., Pavaloaia V.-D. The race for making up the list of emergent smart cities. An Eastern European country's approach // Transformation in Business and Economics, 2015, vol. 14, no. 2A, pp. 529-548.

18. Giffinger R., Fertner C., Kramar H., Kalasek R., Pichler-Milanović N., Meijers E. Smart Cities - Ranking of European Medium-Sized Cities. Vienna: Centre of Regional Science, Vienna University of Technology, 2007. 24 p.

19. Goodspeed R. Smart cities: Moving beyond urban cybernetics to tackle wicked problems // Cambridge Journal of Regions, Economy and Society, 2015, vol. 8, no. 1, pp. 79-92. DOI: 10.1093/cjres/rsu013.

20. Hagerstrand T. Innovation Diffusion as a Spatial Process. Chicago: The University of Chicago Press, 1967. 350 p.

21. Herrschel T. Competitiveness and sustainability: Can "smart city regionalism" square the circle? // Urban Studies, 2013, vol. 50, no. 11, pp. 2332-2348. DOI: $10.1177 / 0042098013478240$.
22. Hollands R.G. Will the real smart city please stand up? Intelligent, progressive or entrepreneurial? // City, 2008, vol. 12, no. 3, pp. 303-320. DOI: $10.1080 / 1360481080247926$.

23. Ingwersen P., Serrano-López A. E. Smart city research 1990-2016 // Scientometrics, 2018, vol. 117, no. 2, pp. 1205-1236. DOI: 10.1007/s11192-018-2901-9.

24. Ivaldi E., Penco L., Isola G., Musso E. Smart sustainable cities and the urban knowledge-based economy: A NUTS3 level analysis // Social Indicators Research, 2020, vol. 150, pp. 45-72. DOI: 10.1007/s11205-020-02292-0.

25. Kim H.-K., Yi M.-S., Shin D.-B. Regional diffusion of smart city service in South Korea investigated by spatial autocorrelation: Focused on safety and urban management // Spatial Information Research, 2017, vol. 25, no. 6, pp. 837-848. DOI: $10.1007 / \mathrm{s} 41324-017-0150-2$.

26. Kitchin R. The timescape of smart cities // Annals of the American Association of Geographers, 2019, vol. 109, no. 3, pp. 775-790. DOI: 10.1080/24694452.2018.1497475.

27. Kourtit K., Nijkamp P. Smart cities in smart space: A regional science perspective // Scienze Regionali, 2018, vol. 17, no. 1, pp. 105-114. DOI: 10.14650/88819.

28. Lu D., Tian Y., Liu V.Y., Zhang Y. The performance of the smart cities in China - A comparative study by means of self-organizing maps and social networks analysis // Sustainability, 2015, vol. 7, no. 6, pp. 76047621. DOI: $10.3390 /$ su7067604.

29. Masik G., Studzińska D. Ewolucia koncepji I badania miasta inteligentnego // Przeglad Geograficzny, 2018, vol. 90, no. 4, pp. 557-571. DOI: 10.7163/PrzG.2018.4.2.

30. McDuie-Ra D., Lai L. Smart cities, backward frontiers: Digital urbanism in India's north-east // Contemporary South Asia, 2019, vol. 27, no. 3, pp. 358-372. DOI: 10.1080/09584935.2019.1647144.

31. Mora L., Bolici R., Deakin M. The first decades of smart city research: A bibliometric analysis // Journal of Urban Technology, 2017, vol. 24, no. 1, pp. 3-27. DOI: 10.1080/10630732.2017.1285123.

32. Morandi C., Rolando A., di Vita S. From Smart City to Smart Region: Digital Services for an Internet of Places. Milan: Springer-Verlag, 2016. 103 p.

33. Nesti G., Graziano P.R. The democratic anchorage of governance networks in smart cities: An empirical assessment // Public Management Review, 2020, vol. 22, no. 5, pp. 648-667. DOI: $10.1080 / 14719037.2019 .1588355$.

34. Neville W. Managing the smart city-state: Singapore approaches the 21st century // New Zealand Geographer, 1999, vol. 55, no. 1, pp. 35-45. DOI: 10.1111/j.17457939.1999.tb01553x.

35. Ogrodnik K. Multi-criteria analysis of smart cities in Poland // Geographia Polonica, 2020, vol. 93, no. 2, pp. 163-181. DOI: 10.7163/GPol.0168.

36. Patrão C., Moura P., de Almeida A.T. Review of smart city assessment tools // Smart Cities, 2020, vol. 3, pp. 1117-1132. DOI: 10.3390/smartcities3040055.

37. Persai P., Katiyar S. K. Development of information evaluation system for smart city planning using geoin- 
formatics techniques // Journal of Indian Society of Remote Sensing, 2018, vol. 46, no. 11, pp. 1881-1891.

38. Peters D. J., Hamideh S., Zarecor K. E., Ghandour M. Using entrepreneurial social infrastructure to understand smart shrinkage in small towns // Journal of Rural Studies, 2018, vol. 64, pp. 39-49. DOI: 10.1016/j.jrurstud.2018.10.001.

39. Picon A. Smart Cities: A Spatialised Intelligence. Chichester: John Wiley \& Sons, 2015. 168 p.

40. Praharaj S., Han H. Building a typology of the 100 smart cities in India // Smart and Sustainable Built Environment, 2019, vol. 8, no. 5, pp. 400-414. DOI: 10.1108/ SASBE-04-2019-0056.

41. Raspotnik A., Grønning R., Herrmann V. A tale of three cities: The concept of smart sustainable cities for the Arctic // Polar Geography, 2020, vol. 43, pp. 64-87. DOI: 10.1080/1088937X.2020.1713546.

42. Roche S. Geographic information science I: Why does a smart city need to be spatially enabled? // Progress in Human Geography, 2014, vol. 38, no. 5, pp. 703-711. DOI: $10.1177 / 0309132513517365$.

43. Ruhlandt R.W.S. The governance of smart cities: A systematic literature review // Cities, 2018, vol. 81, pp. 1-23. DOI: 10.1016/j.cities.2018.02.014.

44. Safransky S. Geographies of algorithmic violence: Redlining the smart city // International Journal of Urban and Regional Research, 2020, vol. 44, pp. 200-218. DOI: 10.1111/1468-2427.12833.
45. Shearmur R., Charron M., Pajevic F. Pourquoi seules les villes sont-elles qualifiées d'intelligentes? Un vocabulaire du biais urbain // Canadian Geographer, 2020, vol. 64, no. 2, pp. 310-322. DOI: 10.1111/cag. 12573.

46. Shelton T., Zook M., Wiig A. The "actually existing smart city" // Cambridge Journal of Regions, Economy and Society, 2015, vol. 8, no. 1, pp. 13-25. DOI: 10.1093/ cjres/rsu026.

47. Slee B. Delivering on the concept of smart villages - in search of an enabling theory // European Countryside, 2019, vol. 11, pp. 634-650. DOI: 10.2478/euco-2019-0035.

48. Vasilić M. Operacionalizacija koncepta "pametnog" grada na primeru Srbije // Sociologija, 2018, vol. 60, no. 2, pp. 518-537. DOI: $10.2298 /$ SOC1802518V.

49. Wathne M.W., Haarstad H. The smart city as mobile policy: Insights on contemporary urbanism // Geoforum, 2020, vol. 108, pp. 130-138. DOI: 10.1016/j. geoforum.2019.12.003.

50. Wiig A. The empty rhetoric of the smart city: From digital inclusion to economic promotion in Philadelphia // Urban Geography, 2016, vol. 37, pp. 535-553. DOI: 10.1080/02723638.2015.1065686.

Конфликт интересов: Автор декларирует отсутствие явных и потенциальных конфликтов интересов, связанных с публикацией настоящей статьи.

Поступила в редакцию 23.03.2021

Принята к публикации 03.09.2021

\title{
Geographical study of "smart cities": priorities, approaches, prospects
}

\author{
V.I. Blanutsa ${ }^{\text {网 }}$ \\ V.B. Sochava Institute of Geography, Siberian Branch of the RAS, Russian Federation \\ (1, Ulan-Batorskaya St., Irkutsk, 664033)
}

\begin{abstract}
The purpose is to identify existing approaches and outline future approaches to the integrated geographical study of "smart cities".

The information base is one domestic and seven international bibliographic databases.

The methods are comparative analysis and the author's algorithm of semantic search based on machine learning.

Results and Discussion. Ninety-one articles were identified. The distribution of articles by country and scale of the study is shown. The analysis of the articles made it possible to identify eight existing approaches: comparative-geographical, spatial-urban, regional, geopolitical, spatial-segregation, temporal-landscape, spatial-diffusion and geosystem. It is assumed that in the future there may be three more approaches: positional-geographical, flow-based and system-based settlement. A relatively small number of articles indicates the stage of formation of the research under consideration. In the future, it is possible to converge all approaches into a single methodology for the geographical study of "smart cities".
\end{abstract}

(C) Blanutsa V.I., 2021

凶 Viktor I. Blanutsa, e-mail: blanutsa@list.ru

The content is available under Creative Commons Attribution 4.0 License. 
Key words: human geography, smart city, comparative analysis, zoning, socio-spatial inequality, spatial diffusion, economic-geographical location.

Funding: The study was carried out at the expense of the state task (registration number of the topic AAAA-A17-117041910166-3).

For citation: Blanutsa V.I. Geographical study of "smart cities": priorities, approaches, prospects. Vestnik Voronezskogo gosudarstvennogo universiteta. Seria: Geografia. Geoekologia, 2021, no. 3, pp. 3-12. (In Russ.) DOI: https://doi.org/10.17308/geo.2021.3/3595

\section{REFERENCES}

1. Baransky N.N. Izbrannye trudy. Stanovlenie ekonomicheskoj geografii [Selected Works. Formation of Economic Geography]. Moscow: Mysl, 1980. 287 p. (In Russ.)

2. Blanutsa V.I. Social'no-ekonomicheskoe rajonirovanie $\mathrm{v}$ epohu bol'shih dannyh [Socio-Economic Zoning in the Era of Big Data]. Moscow: INFRA-M, 2018. 194 p. (In Russ.)

3. Blanutsa V. I. Информационно-сетевая география [Information-Network Geography]. Moscow: INFRA-M, 2019. 243 p. (In Russ.)

4. Blanutsa V.I. Regional'nye ekonomicheskie issledovaniya $\mathrm{s}$ ispol'zovaniem algoritmov iskusstvennogo intellekta: sostoyanie i perspektivy [Regional economic research using artificial intelligence algorithms: State and prospects]. Vestnik Zabajkal'skogo gosudarstvennogo universiteta, 2020, vol. 26, no. 8, pp. 100-111. (In Russ.) DOI: 10.21209/2227-9245-2020-26-8-100-111.

5. Adam S. A critical geographical analysis of the smart city concept - Theoretical background and possible research directions. Ter es Tarsadalom, 2020, vol. 34, no. 2, pp. 88-107. DOI: 10.17649/TET.34.2.3201.

6. Angelidou M. Smart city policies: A spatial approach. Cities, 2014, vol. 41, pp. S3-S11. DOI: 10.1016/j. cities.2014.06.007.

7. Blanutsa V.I. Economic-geographical location: Generalization of conceptual frameworks and generation of new meanings. Geography and Natural Resources, 2015, vol. 36, no. 4, pp. 319-326. DOI: 10.1134/s1875372815040010.

8. Borsekova K., Koróny S., Vaňová A., Vitálišová K. Functionality between the size indicators of smart cities: A research challenge with policy implications. Cities, 2018, vol. 78, pp. 17-26. DOI: 10.1016/j.cities.2018.03.010.

9. Calzada I. Problematizing and politicizing smart city-regions: Is devolution smart? Territorio, 2017, vol. 83, pp. 37-47. DOI: 10.3280/TR2017-083005.

10. Caragliu A., Del Bo C., Nijkamp P. Smart cities in Europe. Journal of Urban Technology, 2011, vol. 18, no. 2, pp. 65-82. DOI: 10.1080/10630732.2011.601117.

11. Caragliu A., Del Bo C. Much ado about something? An appraisal of the relationship between smart city and smart specialization policies. Tijdschrift voor Economische en Sociale Geographie, 2018, vol. 109, no. 1, pp. 129-143. DOI: 10.1111/tesg. 12272.

12. Chen X., Wei L., Zhang H. Spatial and temporal pattern of urban smart development in China and its driving mechanism. Chinese Geographical Science, 2018, vol. 28, no. 4, pp. 584-599. DOI: 10.1007/s11769-018-0976-0.

13. Coletta C., Heaphy L., Kitchin R. From the accidental to articulated smart city: The creation and work of "Smart
Dublin". European Urban and Regional Studies, 2019, vol. 26, no. 4, pp. 349-364. DOI: 10.1177/0969776418785214.

14. De Falco S., Angelidou M., Addie J.-P.D. From the "smart city" to the "smart metropolis"? Building resilience in the urban periphery. European Urban and Regional Studies, 2019, vol. 26, no. 2, pp. 205-223. DOI: $10.1177 / 0969776418783813$.

15. Desdemoustier J., Crutzen N., Giffinger R. Municipalities' understanding of the Smart City concept: An exploratory analysis in Belgium. Technological Forecasting and Social Change, 2019, vol. 142, pp. 129-141. DOI: 10.1016/j. techfore.2018.10.029.

16. Fariniuk T.M.D. Smart cities and the pandemic: Digital technologies on the urban management of Brazilian cities. Revista de Administração Pública, 2020, vol. 54, no. 4, pp. 860-873.

17. Georgescu M., Tugui A., Pavaloaia V.-D. The race for making up the list of emergent smart cities. An Eastern European country's approach. Transformation in Business and Economics, 2015, vol. 14, no. 2A, pp. 529-548.

18. Giffinger R., Fertner C., Kramar H., Kalasek R., Pichler-Milanović N., Meijers E. Smart Cities - Ranking of European Medium-Sized Cities. Vienna: Centre of Regional Science, Vienna University of Technology, 2007. 24 p.

19. Goodspeed R. Smart cities: Moving beyond urban cybernetics to tackle wicked problems. Cambridge Journal of Regions, Economy and Society, 2015, vol. 8, no. 1, pp. 79-92. DOI: 10.1093/cjres/rsu013.

20. Hagerstrand T. Innovation Diffusion as a Spatial Process. Chicago: The University of Chicago Press, 1967. $350 \mathrm{p}$.

21. Herrschel T. Competitiveness and sustainability: Can "smart city regionalism" square the circle? Urban Studies, 2013, vol. 50, no. 11, pp. 2332-2348. DOI: $10.1177 / 0042098013478240$.

22. Hollands R.G. Will the real smart city please stand up? Intelligent, progressive or entrepreneurial? City, 2008, vol.12,no.3,pp.303-320.DOI: 10.1080/1360481080247926.

23. Ingwersen P., Serrano-López A.E. Smart city research 1990-2016. Scientometrics, 2018, vol. 117, no. 2, pp. 1205-1236. DOI: 10.1007/s11192-018-2901-9.

24. Ivaldi E., Penco L., Isola G., Musso E. Smart sustainable cities and the urban knowledge-based economy: A NUTS3 level analysis. Social Indicators Research, 2020, vol. 150, pp. 45-72. DOI: 10.1007/s11205-020-02292-0.

25. Kim H.-K., Yi M.-S., Shin D.-B. Regional diffusion of smart city service in South Korea investigated by spatial autocorrelation: Focused on safety and urban management. Spatial Information Research, 2017, vol. 25, no. 6 , pp. 837-848. DOI: $10.1007 / \mathrm{s} 41324-017-0150-2$. 
26. Kitchin R. The timescape of smart cities. Annals of the American Association of Geographers, 2019, vol. 109, no. 3, pp. 775-790. DOI: 10.1080/24694452.2018.1497475.

27. Kourtit K., Nijkamp P. Smart cities in smart space: A regional science perspective. Scienze Regionali, 2018, vol. 17 , no. 1, pp. 105-114.

28. Lu D., Tian Y., Liu V.Y., Zhang Y. The performance of the smart cities in China - A comparative study by means of self-organizing maps and social networks analysis. Sustainability, 2015, vol. 7, no. 6, pp. 7604-7621. DOI: $10.3390 /$ su7067604.

29. Masik G., Studzińska D. Ewolucia koncepji I badania miasta inteligentnego. Przeglad Geograficzny, 2018, vol. 90, no. 4, pp. 557-571. DOI: 10.7163/PrzG.2018.4.2.

30. McDuie-Ra D., Lai L. Smart cities, backward frontiers: Digital urbanism in India's north-east. Contemporary South Asia, 2019, vol. 27, no. 3, pp. 358-372. DOI: 10.1080 /09584935.2019.1647144.

31. Mora L., Bolici R., Deakin M. The first decades of smart city research: A bibliometric analysis. Journal of Urban Technology, 2017, vol. 24, no. 1, pp. 3-27. DOI: 10.1080/10630732.2017.1285123.

32. Morandi C., Rolando A., di Vita S. From Smart City to Smart Region: Digital Services for an Internet of Places. Milan: Springer-Verlag, 2016. 103 p.

33. Nesti G., Graziano P.R. The democratic anchorage of governance networks in smart cities: An empirical assessment. Public Management Review, 2020, vol. 22, no. 5, pp. 648-667. DOI: 10.1080/14719037.2019.1588355.

34. Neville W. Managing the smart city-state: Singapore approaches the 21 st century. New Zealand Geographer, 1999, vol. 55, no. 1, pp. 35-45. DOI: 10.1111/j.17457939.1999.tb01553x.

35. Ogrodnik K. Multi-criteria analysis of smart cities in Poland. Geographia Polonica, 2020, vol. 93, no. 2, pp. 163-181. DOI: 10.7163/GPol.0168.

36. Patrão C., Moura P., de Almeida A.T. Review of smart city assessment tools. Smart Cities, 2020, vol. 3, pp. 1117-1132. DOI: 10.3390/smartcities3040055.

37. Persai P., Katiyar S. K. Development of information evaluation system for smart city planning using geoinformatics techniques. Journal of Indian Society of Remote Sensing, 2018, vol. 46, no. 11, pp. 1881-1891.

38. Peters D.J., Hamideh S., Zarecor K.E., Ghandour M. Using entrepreneurial social infrastructure to understand smart shrinkage in small towns. Journal of Rural Studies, 2018, vol. 64, pp. 39-49. DOI: 10.1016/j. jrurstud.2018.10.001.

\section{Блануца Виктор Иванович}

доктор географических наук, ведущий научный сотрудник Института географии им. В.Б. Сочавы CO PAH, г. Иркутск, Российская Федерация, ORCID: 0000-0003-3958-216X, e-mail: blanutsa@list.ru
39. Picon A. Smart Cities: A Spatialised Intelligence. Chichester: John Wiley \& Sons, 2015. 168 p.

40. Praharaj S., Han H. Building a typology of the 100 smart cities in India. Smart and Sustainable Built Environment, 2019, vol. 8, no. 5, pp. 400-414. DOI: 10.1108/SASBE-04-2019-0056.

41. Raspotnik A., Grønning R., Herrmann V. A tale of three cities: The concept of smart sustainable cities for the Arctic. Polar Geography, 2020, vol. 43, pp. 64-87. DOI: 10.1080/1088937X.2020.1713546.

42. Roche S. Geographic information science I: Why does a smart city need to be spatially enabled? Progress in Human Geography, 2014, vol. 38, no. 5, pp. 703-711. DOI: $10.1177 / 0309132513517365$.

43. Ruhlandt R.W.S. The governance of smart cities: A systematic literature review. Cities, 2018, vol. 81, pp. 1-23. DOI: 10.1016/j.cities.2018.02.014.

44. Safransky S. Geographies of algorithmic violence: Redlining the smart city. International Journal of Urban and Regional Research, 2020, vol. 44, pp. 200-218. DOI: 10.1111/1468-2427.12833.

45. Shearmur R., Charron M., Pajevic F. Pourquoi seules les villes sont-elles qualifiées d'intelligentes? Un vocabulaire du biais urbain. Canadian Geographer, 2020, vol. 64 , no. 2 , pp. 310-322. DOI: 10.1111/cag. 12573 .

46. Shelton T., Zook M., Wiig A. The "actually existing smart city". Cambridge Journal of Regions, Economy and Society, 2015, vol. 8, no. 1, pp. 13-25. DOI: 10.1093/ cjres/rsu026.

47. Slee B. Delivering on the concept of smart villages - in search of an enabling theory. European Countryside, 2019, vol. 11, pp. 634-650. DOI: 10.2478/euco-2019-0035.

48. Vasilić M. Operacionalizacija koncepta "pametnog" grada na primeru Srbije. Sociologija, 2018, vol. 60, no. 2, pp. 518-537. DOI: 10.2298/SOC1802518V.

49. Wathne M.W., Haarstad H. The smart city as mobile policy: Insights on contemporary urbanism. Geoforum, 2020, vol. 108, pp. 130-138. DOI: 10.1016/j.geoforum.2019.12.003.

50. Wiig A. The empty rhetoric of the smart city: From digital inclusion to economic promotion in Philadelphia. Urban Geography, 2016, vol. 37, pp. 535-553. DOI: $10.1080 / 02723638.2015 .1065686$.

Conflict of interest: The author declares no obvious and potential conflicts of interest to the publication of this article.

Received: 23.03.2021

Accepted: 03.09.2021

Viktor I. Blanutsa

Doc. Sci. (Geogr.), Leading Researcher of the V.B. Sochava Institute of Geography, Siberian Branch of the RAS, Irkutsk, Russian Federation, ORCID: 0000-0003-3958216X, e-mail: blanutsa@list.ru 